\title{
DETERMINAÇÃO DE FOSFATO EM REFRIGERANTES UTILIZANDO UM SCANNER DE MESA E ANÁLISE AUTOMATIZADA DE DADOS: UM EXEMPLO DIDÁTICO PARA ENSINO DE QUÍMICA
}

\author{
Heloisa Colzani ${ }^{\text {a }}$ Quethelen Elizabeth Araujo Garcez Rodrigues ${ }^{\mathrm{a}}$, Claudia Fogaça ${ }^{\mathrm{b}}$, Jane Mary Lafayette Neves Gelinski', \\ Edenir R. Pereira-Filho ${ }^{c}$ e Endler Marcel Borges ${ }^{\mathrm{a}, \mathrm{b}, *}$ \\ aDepartamento de Química, Universidade Regional de Blumenau, Campus 1, Rua Antônio da Veiga, 140, Victor Konder, $89012-$ \\ 900 Blumenau - SC, Brasil \\ ${ }^{b}$ Núcleo Biotecnológico, Universidade do Oeste de Santa Catarina, Rua Paese, 198, Bairro Universitário-Bloco K, 89560-000 \\ Videira - SC, Brasil \\ 'Departamento de Química, Universidade Federal de São Carlos, Rodovia Washington Luiz (SP 340), km 235, CP 676, 13565-905 \\ São Carlos - SP, Brasil
}

Recebido em 19/10/2016; aceito em 31/01/2017; publicado na web em 24/03/2017

\begin{abstract}
PHOSPHATE COLORIMETRIC ANALYSIS USING A DESKTOP SCANNER AND AUTOMATED DIGITAL IMAGE ANALYSIS: A DIDATICAL EXAMPLE TO TEACH COLORIMETRIC ANALYSES. We propose here the quantification of the phosphorus content in cola drinks by molybdenum blue method. Molybdenum blue method was carried out in the wells of a microplate, where method miniaturization represents reduction in reagents required, improved safety, reduced waste stream, and increased sample throughput. The phosphate concentration was determinate using scanned images of the microplate. Extraction of RGB values was carried out using ImageJ, the pugling Readplate was used allowing extraction of RGB values of all wells simultaneously. A Microsoft Excel spreadsheet is afforded in Google Drive, it organize data taken from ImageJ to afford calibrations curves and phosphate concentration in cola drinks. Calibration curves were prepared at $500-2500 \mu \mathrm{g} \mathrm{L}^{-1}$ of $\mathrm{P}$ with good linearity $(\mathrm{R}>0.95)$. Phosphate concentrations in cola drinks determinate using digital images method are statistically equivalent to concentrations determined using a spectroscopic method at a $95 \%$ confidence interval.
\end{abstract}

Keywords: RGB; digital image; ImageJ; phosphate.

\section{INTRODUÇÃO}

Nas últimas décadas, vários métodos colorimétricos foram adaptados para microplacas, em especial, na área clínica e em análises ambientais. As vantagens de adaptar os métodos tradicionais são a redução no uso de reagentes e consequente menor geração de resíduos químicos. No entanto, o uso de técnicas de microescala empregando microplacas, em sala de aula, é provavelmente limitada pelo custo elevado (ordem de US\$ 10.000) dos leitores de microplacas. ${ }^{1}$

Normalmente, as imagens digitais utilizam o sistema Red, Green e Blue (RGB) para a definição de cores. Nesse sistema, cada tom de cor é definido por três canais: $R$ (vermelho), $G$ (verde) e B (azul), que variam como índices inteiros entre 0 e 255 , onde o 0 corresponde à cor preta e o 255 corresponde ao R ou G ou B puros. Desta forma, no sistema RGB, um tom de cor corresponde a um ponto em um espaço tridimensional formado pelos eixos $\mathrm{R}, \mathrm{G}$ e B, sendo possível a geração de 16 milhões de cores diferentes $\left(256^{3}\right) .^{2}$

A absorbância é definida de acordo com a Equação 1. A potência de um feixe $(\mathrm{P})$, transmitida através de uma célula com a solução do analito, é comparada com a potência que atravessa uma célula idêntica contendo somente o solvente ou o branco dos reagentes $\left(\mathrm{P}_{0}\right)$. De acordo com a lei Beer-Lambert, a absorbância (A) é proporcional a concentração do analito. ${ }^{1}$

$$
\boldsymbol{A}=\log \left(\frac{\boldsymbol{P}}{\boldsymbol{P}_{0}}\right)
$$

Kohl et al. ${ }^{3}$ desenvolveram uma prática de ensino que mostrou que o princípio da absorbância pode ser facilmente demonstrada utilizando

*e-mail: marcelborgesb@gmail.com soluções coloridas e análises de imagens digitais. A prática de ensino consistia em fotografar um conjunto de cubetas contendo corante amarelo em diferentes concentrações e uma cubeta contendo apenas água destilada. Os valores de $\mathrm{G}$ foram obtidos para as cubetas que continham o corante amarelo e $\mathrm{G}_{0}$ foi obtido da cubeta que continha apenas água destilada. Posteriormente, estes valores foram utilizados para determinar a absorbância e preparar curvas analíticas de calibração.

Atualmente, o processamento de imagens digitais tem atraído grande atenção como uma ferramenta analítica para a análise colorimétrica devido a sua versatilidade, compreendendo procedimentos simples, rápidos e de baixo custo. Existem vários exemplos relevantes descritos na literatura, tais como para a determinação de nitrito em alimentos, amostras ambientais e amostras clínicas, ${ }^{4}$ na determinação do diclofenaco sódico, dipirona sódica e gluconato de cálcio em drogas injetáveis, ${ }^{5}$ análise de chás, ${ }^{6}$ quantificação de etanol em bebidas, ${ }^{7}$ e quantificação de sulfito em bebidas. ${ }^{8}$ Além disso, o uso de imagens digitais pode ser uma alternativa para ensaios clínicos, como demonstrado recentemente para creatinina ${ }^{9}$ e determinação de glucose, ${ }^{10}$ alanina aminotransferase. ${ }^{11}$ Por sua vez, as imagens digitais também podem ser utilizadas para controle de qualidade. Por exemplo, Santos et al. ${ }^{12,13}$ utilizaram imagens digitais obtidas com um scanner de mesa para verificar adulterações do leite.

Recentemente, Christodouleas et al. ${ }^{14}$ mostraram que um scanner de mesa pode substituir um leitor de microplacas, fazendo a quantificação de diversos corantes e leituras de placas de teste ELISA (ensaio de imunoabsorção enzimática, Enzyme-Linked Immunosorbent Assay).

No intuito de associar o cotidiano do aluno de graduação com as práticas de laboratório, foi proposta a determinação da concentração de fosfato em refrigerantes utilizando o método do azul de molibdênio. ${ }^{15,16}$ 
O método do azul de molibdênio consiste na formação de um complexo entre o ânion fosfato com um composto de molibdato em solução ácida (Reação 1).

Reação 1: $\mathrm{PO}_{4}{ }^{3-}+12 \mathrm{MoO}_{4}{ }^{2-}+27 \mathrm{H}^{+} \leftrightarrows \mathrm{H}_{3} \mathrm{PO}_{4}\left(\mathrm{MoO}_{3}\right)_{12}+12 \mathrm{H}_{2} \mathrm{O}$

O complexo fosfato molibdênio hexavalente incolor é reduzido a uma forma pentavalente azul por um agente redutor (ácido ascórbico) em meio ácido (Reação 2).

Reação 2: $\mathrm{H}_{3} \mathrm{PMo}(\mathrm{VI})_{12} \mathrm{O}_{40}+$ Redutor $\leftrightarrows\left[\mathrm{H}_{4} \mathrm{PMo}(\mathrm{VI})_{8} \mathrm{Mo}(\mathrm{V})_{4} \mathrm{O}_{40}\right]^{3-}$

A intensidade da cor azul é medida espectrofotometricamente a 600,650 e $800 \mathrm{~nm}^{17}$

Cola é uma bebida carbonatada doce (denominada refrigerante ou gasosa), e normalmente contém corante de caramelo, cafeína, mistura de baunilha, canela e sabores cítricos. O nome vem da castanha de cola que originalmente é usada para obter a cafeína. ${ }^{18}$

Bebidas de cola são bastante consumidas e populares entre os estudantes. Muita atenção está sendo dada para possíveis consequências do consumo excessivo para a saúde humana. Estudos vem sendo feitos para avaliar o efeito destas bebidas na erosão dentária, ${ }^{15}$, na ingestão de altos níveis de cafeína que podem causar hipertensão, reações alérgicas e distúrbios gastrointestinais. ${ }^{19,20}$ Além disso, estas bebidas podem acaretar efeitos adversos no metabolismo do cálcio devido ao seu alto teor de ácido fosfórico, que combinado com a baixa ingestão de cálcio na dieta pode aumentar o risco de doenças ósseas..$^{21,22}$

Desta forma, o presente estudo utiliza o método do azul de molibdênio para determinar a concentração de fosfato em refrigerantes a base de cola. As reações são feitas diretamente nos poços das microplacas de cultura celular (resultando em baixo consumo de reagentes) e a concentração de fosfato é determinada utilizando imagens digitais. Os valores de RGB foram extraídos simultaneamente de todos os poços utilizando um plugin do programa ImageJ. . $^{232}$

\section{PARTE EXPERIMENTAL}

\section{Reagentes}

No desenvolvimento da parte experimental foram utilizados os seguintes reagentes: Tartarato de antimônio e potássio, $\mathrm{C}_{4} \mathrm{H}_{4} \mathrm{KO}_{7} \mathrm{Sb}_{1 / 2} \mathrm{H}_{2} \mathrm{O}, 99,8 \%$, fosfato de potássio monóbasico anidro, $\mathrm{KH}_{2} \mathrm{PO}_{4}, 99 \%$, molibdato de amônio, $\mathrm{NH}_{4} \mathrm{Mo}_{7} \mathrm{O}_{24} 4 \mathrm{H}_{2} \mathrm{O}, 81 \%$, ácido $\mathrm{L}(+)$ Ascórbico, $\mathrm{C}_{6} \mathrm{H}_{8} \mathrm{O}_{6}, 99 \%$. Todos os reagentes são da marca Vetec (Rio de Janeiro, Brasil).

\section{Equipamentos}

As imagens foram obtidas com um scanner HP Scanjet G2710 e o software do scanner era o HP Scanning. A concentração de fosfato também foi determinada utilizando-se um leitor de microplacas Tecan Infinite 200 PRO (TECAN, Suiça).

\section{Soluções}

Para a aquisição das imagens foram preparadas as soluções listadas a seguir:

A: $15 \mathrm{ml}$ de ácido sulfúrico concentrado em $100 \mathrm{~mL}$ de água deionizada.

B: $0,3 \mathrm{~g}$ de tartarato de antimônio e potássio em $100 \mathrm{~mL}$ de água deionizada.

C: $1,2 \mathrm{~g}$ de molibdato de amônio em $100 \mathrm{~mL}$ de água deionizada.

D: $1,76 \mathrm{~g}$ de ácido ascórbico em $100 \mathrm{~mL}$ de água deionizada.
Para a preparação do reagente misto as soluções A a D foram misturadas nas seguintes proporções e ordem: $5 \mathrm{~mL}$ da solução A $+1 \mathrm{~mL}$ da solução $\mathrm{B}+5 \mathrm{~mL}$ da solução $\mathrm{C}+5 \mathrm{~mL}$ da solução $\mathrm{D}$.

Solução estoque de $\mathrm{KH}_{2} \mathrm{PO}_{4}$ : $220 \mathrm{mg}$ de $\mathrm{KH}_{2} \mathrm{PO}_{4}$ diluído em 1 $\mathrm{L}$ de água deionizada que representa $50 \mathrm{mg} \mathrm{L}^{-1} \mathrm{de} \mathrm{P}$ (fósforo). Por exemplo, $220 \mathrm{mg} \mathrm{L}^{-1}$ de $\mathrm{KH}_{2} \mathrm{PO}_{4}$ representam 1,62 $\mathrm{mmol} \mathrm{L}^{-1}$ de $\mathrm{KH}_{2} \mathrm{PO}_{4}$, visto que, a massa molecular do $\mathrm{KH}_{2} \mathrm{PO}_{4}$ é $136,09 \mathrm{~g} \mathrm{~mol}^{-1}$ (220 $\left.\mathrm{mg} \mathrm{L}^{-1} / 136,09 \mathrm{~g} \mathrm{~mol}^{-1}=1,62 \mathrm{mmol} \mathrm{L}^{-1}\right)$, como a massa molecular do fósforo (P) é de 30,97 $\mathrm{g} \mathrm{mol}^{-1}$, a concentração de P na solução em mg é $1,62 \mathrm{mmol} \mathrm{L}^{-1}$ x $30,97 \mathrm{~g} \mathrm{~mol}^{-1}=50 \mathrm{mg} \mathrm{L}^{-1}$

Na preparação das soluções padrões para estabelecer as curvas de calibração foi empregada uma solução de trabalho: $5 \mathrm{~mL}$ da solução estoque de $\mathrm{KH}_{2} \mathrm{PO}_{4}$ em $100 \mathrm{~mL}$ de água deionizada (2,5 $\mathrm{mg} \mathrm{L}^{-1}$ de $\left.\mathrm{P}\right)$.

\section{CURVA DE CALIBRAÇÃO}

A curva de calibração foi preparada colocando $2,4,6,8 \mathrm{~mL}$ da solução de trabalho de $\mathrm{KH}_{2} \mathrm{PO}_{4}$ em balões de $10 \mathrm{~mL}$, com o auxílio de uma bureta de $25 \mathrm{~mL}$, e completando o volume até o menisco com água deionizada. O último ponto da curva de calibração era a própria solução de trabalho. A concentração de fosfato foi expressa como $\mu \mathrm{g} \mathrm{L} \mathrm{L}^{-1}$ de fósforo. A concentração de cada ponto da curva foi calculada utilizando a Equação 2, em que a concentração de solução de trabalho $\left(2,5 \mathrm{mg} \mathrm{L}^{-1}\right)$ é $\mathrm{C}_{2}, \mathrm{~V}_{2}$ é o volume da solução de trabalho adicionado ao balão volumétrico de $10 \mathrm{~mL}$ e $\mathrm{V}_{1}$ é $10 \mathrm{~mL}$. Portanto, a concentração de cada ponto da curva analítica foi de 500, 1000, 1500,2000 e $2500 \mu \mathrm{g} \mathrm{L}{ }^{-1}$.

$$
\mathrm{C}_{1}=\mathrm{C}_{2} \mathrm{~V}_{2} / \mathrm{V}_{1}
$$

\section{Preparo das soluções de refrigerante.}

Devido à elevada concentração de fosfato nos refrigerantes, estes foram diluídos da seguinte forma: $1 \mathrm{~mL}$ de refrigerante foi diluído em um balão de $100 \mathrm{~mL}$ (100 vezes diluída) e $1 \mathrm{ml}$ de refrigerante foi diluído em um balão de $50 \mathrm{~mL}$ (50 vezes diluída).

Os refrigerantes utilizados foram Pepsi, Coca Zero, Coca-Cola e Guaraná Antártica Black.

\section{Método do Azul de molibdênio.}

Duzentos $\mu \mathrm{L}$ de cada amostra de refrigerante diluída foram colocadas em 4 poços da microplaca. Na Figura 1, linhas de E a H e colunas 1 a 5 correspondem aos poços da microplaca que contém as soluções da curva de calibração. Em seguida, $200 \mu \mathrm{L}$ de cada solução de refrigerante foram adicionados a 4 poços da placa, na Figura 1, linhas A a D e colunas 4 a 11. Cada refrigerante foi preparado com duas diluições, 1:50 e 1:100. Por fim, adicionou-se $50 \mu \mathrm{L}$ do reagente misto a cada poço da placa. Após 15 minutos, foram realizadas as leituras em $650 \mathrm{~nm}$ no leitor de microplacas. Posteriormente, as placas foram colocadas em um scanner e as imagens obtidas em 300 pontos por polegada (dots per inch, dpi).

\section{Obtenção das imagens digitais}

As imagens foram obtidas colocando as microplacas em um scanner HP Scanjet G2710, cobertas com um fundo branco, por exemplo, folha branca de EVA (etil, vinil e acetato). As imagens foram obtidas com 300 dpi no formato Joint Photographics Experts Group (JPEG). As opções de correção automática da imagem pelo software foram desabilitadas e o software do scanner era o HP Scanning. 


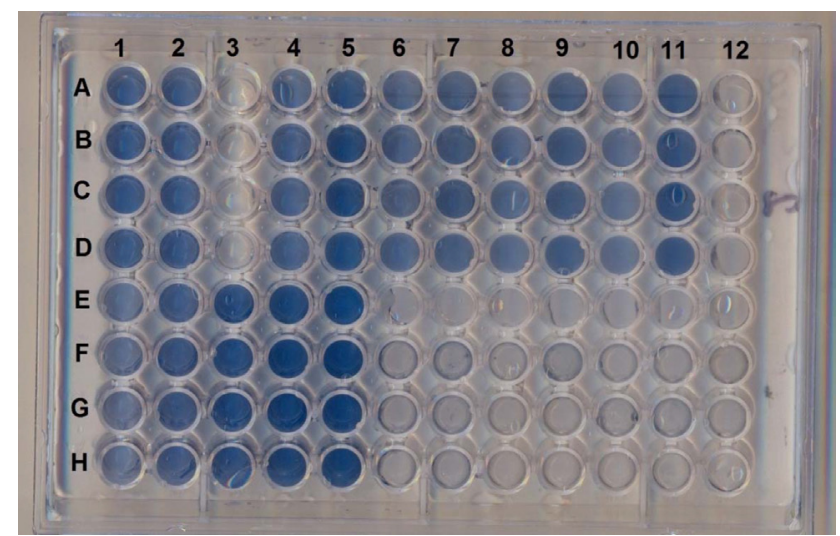

Figura 1. Imagem obtida com scanner de mesa da microplaca frente a um fundo branco

\section{Obtenção dos valores de RGB}

Os valores de RGB foram extraídos das imagens utilizando o software ImageJ, software de uso livre..$^{23}$

Os valores de cada canal (RGB) de todos os poços da microplaca pode ser extraído simultaneamente utilizando o plugin "ReadPalte" do ImageJ.

Por exemplo, para obter os valores R na Figura 1, no ImageJ, clique em Plugins e depois em ReadPlate. Ao clicar em ReadPlate, a janela Measureme... aparecerá. Na janela Measureme... (Figura 2), basta selecionar o canal (Channel) que se deseja extrair. Ao selecionar o canal e clicar em OK, a janela Grid Parameters aparecerá (Figura 3).

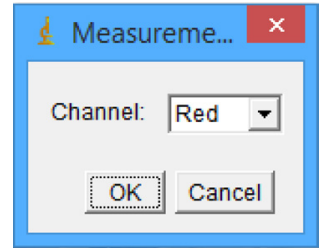

Figura 2. Janela Measureme.... Seleção da canal que se deseja extrair de uma micro placa

Na janela Grid Parameters (Figura 3), em Number of columns, seleciona-se o número de colunas da placa, sendo permitidas no máximo 12 colunas. Em Number of Rows, seleciona-se o número de linhas, sendo permitidas no máximo 8 linhas (de A até $\mathrm{H}$ ).

Em Center of Well A1:X e Center of Well A1:Y, são colocadas as coordenadas em X e Y do primeiro poço A1 (Figura 1). Em Center of Well H12:X e Center of Well H12:Y são colocadas as coordenadas em X e Y do último poço H12 (Figura 1).

No imageJ, as coordenadas dos poços A1 e H12 podem ser determinadas posicionando o cursor sobre a micro placa.

Circle size é o diâmetro do círculo, na placa da onde são extraídos os valores de RGB. Neste trabalho utilizou-se 20 pixel.

Na janela Grid Parameters, após clicar em OK, aparecerá uma janela chamada "Results" contendo (Figura 4), na seguinte ordem, as informações: nome, área, mediana, mínimo, máximo, linha, coluna. Na janela Results, clique "Ctrl + A" para selecionar todos os valores, em seguida clique em "Ctrl + C" para copiar estes valores e para colar estes valores no Microsoft Excel, clique em "Ctrl + V".

Depois de extrair os valores de RGB e exportar os dados para o Microsoft Excel, os valores de R, G e B podem ser exportados para a Tabela 1S, do Microsoft Excel, disponível em um link Google drive..$^{25}$ Na Planilha do Microsoft Excel, os valores de R, G e B foram copiados para as planilhas 1,2 e 3 respectivamente. Nestas planilhas, a média

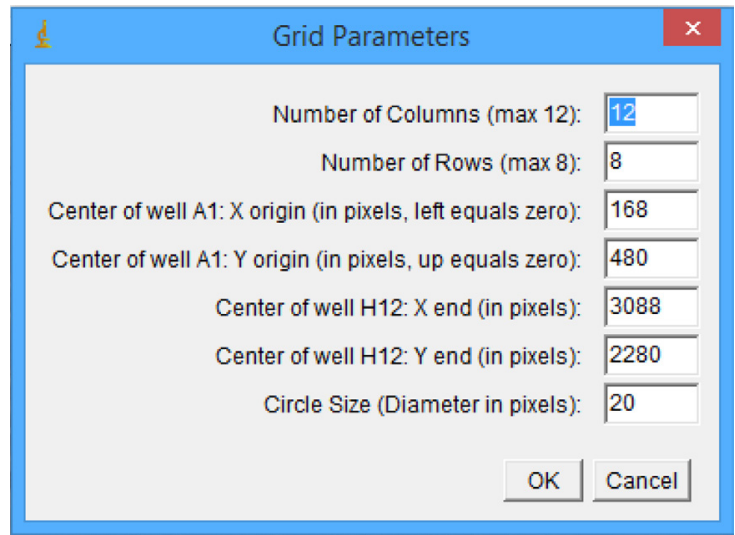

Figura 3. Janela Grid Parameters. Seleção das coordenadas dos poços da microplaca

\begin{tabular}{|c|c|c|c|c|c|c|c|c|}
\hline \multicolumn{9}{|c|}{ File Edit Font Results } \\
\hline & & Area & Mean & Min & $\operatorname{Max}$ & Row & Column & Absorbance \\
\hline 1 & Figura 1.jpg & 316 & 61.649 & 49 & 70 & $\mathrm{~A}$ & 1 & 0.617 \\
\hline 2 & Figura 1.jpg & 316 & 67.304 & 58 & 76 & $\mathrm{~A}$ & 2 & 0.579 \\
\hline 3 & Figura 1.jpg & 316 & 157.918 & 140 & 180 & $\mathrm{~A}$ & 3 & 0.208 \\
\hline 4 & Figura 1.jpg & 316 & 73.915 & 46 & 97 & $\mathrm{~A}$ & 4 & 0.538 \\
\hline 5 & Figura 1.jpg & 316 & 49.883 & 38 & 59 & $\mathrm{~A}$ & 5 & 0.709 \\
\hline 6 & Figura 1.jpg & 316 & 80.835 & 73 & 90 & $\mathrm{~A}$ & 6 & 0.499 \\
\hline 7 & Figura 1.jpg & 316 & 75.044 & 67 & 84 & $\mathrm{~A}$ & 7 & 0.531 \\
\hline
\end{tabular}

Figura 4. Janela results. Janela que mostra os valores de RGB para cada poço da microplaca

dos valores está na coluna $\mathrm{D}$, os valores das médias de $\mathrm{R}, \mathrm{G}$ e $\mathrm{B}$ foram exportados para as colunas $\mathrm{A}, \mathrm{B}$ e $\mathrm{C}$, respectivamente, da planilha 4 .

Na planilha 4, os valores de R, G e B de cada poço são rearranjados de maneira a fornecer as curvas analíticas e a concentração de fosfato em cada um dos refrigerantes utilizando as equações (3-5). As equações de 3 a 6 são as absorbâncias para cada um dos canais RGB, a Equação 6 é a distância euclidiana.

$$
\begin{gathered}
A=-\log \left(\frac{R}{R_{o}}\right)=A \\
A=-\log \left(\frac{G}{G_{o}}\right)=A \\
A=-\log \left(\frac{B}{B_{o}}\right)=A \\
{[v]=\sqrt{\left(R-R_{0}\right)^{2}+\left(G-G_{0}\right)^{2}+\left(B-B_{0}\right)^{2}}}
\end{gathered}
$$

Na planilha 4, os valores extraídos de R, G e B são transferidos para as colunas R, S, T e U, a média (m) dos valores dos quatro poços que contém a curva de calibração são colocados na coluna $\mathrm{N}, \mathrm{o}$ desvio padrão (s) destes quatro valores na coluna $\mathrm{O}$ e o intervalo de confiança na coluna $\mathrm{Q}$, o intervalo de confiança foi calculado com a Equação 7, em que n é o número de poços $(\mathrm{n}=4)$, t é o valor do $\mathrm{t}$ de Student, em um intervalo de confiança de $95 \%$ para três graus de liberdade $(\mathrm{t}=3,18)$, e s é o desvio padrão dos valores de RGB em cada poço. Como é ilustrado na Figura 5.

$$
m \pm \frac{t s}{\sqrt{n}}
$$

Nas colunas H-V, a curva analítica obtida com a Equação 3 está 


\begin{tabular}{|c|c|c|c|c|c|c|c|c|c|c|}
\hline K & $\mathrm{L}$ & M & $\mathrm{N}$ & $\mathrm{O}$ & $\mathrm{P}$ & Q & $\mathrm{R}$ & $\mathrm{s}$ & $\mathrm{T}$ & $\mathrm{U}$ \\
\hline & $\mu \mathrm{g} / \mathrm{L}$ & & $\mathrm{m}$ & s & Desvio padrão relativo & intervalo de confiança & & & & \\
\hline & 500 & 0.0401 & 131 & 5 & 4 & 7.7 & 123.8 & 134.1 & 133.4 & 132.9 \\
\hline & 1000 & 0.1790 & 95 & 7 & 8 & 11.6 & 86.1 & 92.6 & 99.6 & 102.4 \\
\hline & 1500 & 0.2835 & 75 & 6 & 8 & 9.9 & 74.8 & 69.4 & 71.6 & 83.5 \\
\hline & 2000 & 0.5012 & 45 & 6 & 14 & 9.9 & 42.5 & 40.1 & 44.4 & 54.3 \\
\hline & 2501 & 0.6815 & 30 & 10 & 33 & 15.9 & 16.3 & 30.8 & 32.2 & 40.4 \\
\hline & & & 144 & 7 & $5^{\prime}$ & 6.0 & 134.1 & 142.7 & 138.6 & 154.2 \\
\hline & & & & & & 0.0 & 136.2 & 144.8 & 148.0 & 151.3 \\
\hline
\end{tabular}

Figura 5. Tabela $1 S$ disponível no Google Drive, utilizada para converter os valores de RGB extraídos da Figura 1 nas curvas analíticas de calibração

entre as linhas 2 a 7. A curva analítica obtida com a equação 4 está entre as linhas 27 a 33 . A curva analítica obtida com a equação 5 está entre as linhas 51 a 57. A curva analítica obtida com a equação 6 está entre as linhas 74 a 79. A absorbância e [v] de cada amostra de refrigerante, assim como suas respectivas concentrações que foram determinadas utilizando-se as Equações 1 a 4 estão entre as colunas $\mathrm{G}$ a Q e linhas 118 a 129, 132 a 142, 145 a 155 e 158 a 168, respectivamente. A concentração de fósforo em cada refrigerante, determinada com as equações de 1 a 4, estão nas colunas $\mathrm{H}$ a L, respectivamente, nas linhas 174 a 181 .

A Figura 6 mostra como a concentração de P é calculada em cada um dos refrigerantes. A coluna F mostra que estas são as concentrações calculadas com a equação 3 , utilizando os valores de R. Na coluna $\mathrm{G}$ estão os nomes das amostras. Na coluna H estão os fatores de diluição de cada refrigerante. Na coluna J estão as absorbâncias de cada um dos refrigerantes utilizando-se a equação 3. Na coluna $\mathrm{K}$ está representada a concentração de $\mathrm{P}\left(\mu \mathrm{g} \mathrm{L}^{-1}\right)$. Na coluna $\mathrm{L}$ as concentrações determinadas para cada refrigerante após multiplicação por seus respectivos fatores de diluição, por exemplo, um refrigerante que foi diluído 100 vezes, teve a sua concentração determinada na coluna K e este valor foi multiplicado por 100 na coluna L. Na Linha 119, P é o coeficiente angular da equação 3 e Q é o coeficiente linear da equação 1 .

\section{RESULTADOS E DISCUSSÃO}

Existem práticas de ensino descritas na literatura que utilizaram imagens digitais para realizar determinações colorimétricas ${ }^{25-27}$ e de $\mathrm{pH} .{ }^{28,29}$ Nestas práticas de ensino, os valores de RGB foram extraídos utilizando softwares como r-Project ${ }^{25,27}$ Octave e Matlab. 28,29

Quando este experimento foi testado com alunos de graduação em engenharia química, no segundo semestre de 2016, poucos estavam acostumados com este tipo de software, especialmente nos dois primeiros anos de graduação, e se mostram desconfortáveis em trabalhar com os mesmos. Além disto, com estes softwares é necessário recortar as imagens de onde deseja-se extrair os valores de RGB, o que pode ser um procedimento demorado, visto que teríamos de recortar 52 poços separadamente e salvar cada um com um nome específico.

Inicialmente, neste trabalho, os valores de RGB foram extraídos utilizando o r-project, para tanto, pequenos quadrados de 80x80 eram recortados de cada poço da placa e salvos em uma pasta. Posteriormente, os valores de RGB eram extraídos utilizando o r-project.

Devido à dificuldade relatada pelos alunos, foi implementada uma nova metodologia para extrair os valores de RGB das imagens digitais. A alternativa encontrada foi o plugin ReadPlate no ImageJ, que permitiu que os valores de RGB fossem extraídos diretamente das imagens das microplacas em menos de cinco minutos. Importar os valores R, G e B para a Tabela $1 \mathrm{~S}$, disponível no Google drive, ${ }^{25}$ permitiu uma fácil organização dos dados e uma visualização imediata das curvas de calibração, bem como a determinação das concentrações de fosfato nos refrigerantes.

Kohl et al. ${ }^{3}$ demonstram que uma solução contendo um corante amarelo (Bio-Tek ELx808) é amarela porque o complexo absorve o azul da radiação branca que penetra no frasco e transmite o componente amarelo de forma inalterada. Assim, em uma determinação colorimétrica de corante amarelo, o máximo de variação na absorbância, com a concentração, ocorre com a radiação azul. A variação da absorbância com a radiação amarela é desprezível e a radiação empregada em uma análise colorimétrica deve ser a cor complementar da solução do analito. Desta forma, os valores de R e G extraídos de soluções com diferentes concentrações de corante amarelo se mostraram praticamente constantes, enquanto os valores B apresentaram variações significativas em função da concentração do corante. ${ }^{3}$

Neste estudo, o fosfato é determinado como um complexo de coloração azul, ou seja, este absorve a radiação amarela da luz branca e transmite o componente azul. Por esta razão, os métodos analíticos descritos na literatura fazem a leitura da absorbância em 650, 660 e 880 nm. $^{17}$

No sistema RGB, vermelho, verde e azul representam a luz nas regiões de 600-700 nm, 500-600 nm e 400-500 nm, respectivamente. Portanto, devido à coloração azul do complexo, espera-se que os

\begin{tabular}{|c|c|c|c|c|c|c|c|c|c|c|c|c|}
\hline 4 & $\mathrm{~F}$ & G & $\mathrm{H}$ & 1 & J & $\mathrm{K}$ & L & $M$ & $\mathrm{~N}$ & $\mathrm{O}$ & $\mathrm{P}$ & $\mathrm{Q}$ \\
\hline \multicolumn{13}{|l|}{117} \\
\hline \multicolumn{13}{|l|}{118} \\
\hline 119 & & & & & & & & & & & 0.000320926 & -0.14444734 \\
\hline 120 & & & & & & & & & & & $2.31235 \mathrm{E}-05$ & 0.03835515 \\
\hline 121 & & & & & & & & & & & 0.984664221 & 0.03657020 \\
\hline 122 & & pepsi & 100 & 79.65825 & 0.25633 & 1249 & 124883 & & & & & \\
\hline 123 & & pepsi & 50 & 24.4375 & 0.76951 & 2848 & 142393 & & & & & \\
\hline 124 & & coca Zero & 100 & 92.46825 & 0.19157 & 1047 & 104703 & & & & & \\
\hline 125 & & coca Zero & 50 & 58.7745 & 0.38837 & 1660 & 83013 & & & & & \\
\hline 126 & & coca & 100 & 67.364 & 0.32914 & 1476 & 147568 & & & & & \\
\hline 127 & & coca & 50 & 14.32925 & 1.00134 & 3570 & 178513 & & & & & \\
\hline 128 & & \begin{tabular}{|l} 
black \\
\end{tabular} & 100 & 73.93525 & 0.28871 & 1350 & 134972 & & & & & \\
\hline 129 & & black & 50 & 11.754 & 1.08738 & 3838 & 191917 & & & & & \\
\hline
\end{tabular}

Figura 6. Cálculo da concentração de $P\left(\mu g L^{-1}\right)$ em cada refrigerante 
valores B extraídos das imagens digitais variem pouco, em função da concentração de fosfato, e que os valores de $\mathrm{R}$ e $\mathrm{G}$ diminuem em função do aumento da concentração de fosfato.

O comportamento esperado foi observado na prática. A Tabela 1 mostra os valores de RGB para cada um dos pontos da curva analítica, como se esperava, os valores $R$ apresentam a maior variação em função da concentração de fosfato, os valores de $G$ variaram menos em função da concentração de fosfato que os valores de $\mathrm{R}$, enquanto os valores de $\mathrm{B}$ variaram pouco em função da concentração de fosfato.

Tabela 1. Valores de RGB para cada um dos pontos curva analítica, média \pm desvio padrão. O desvio padrão foi calculado para os quatro valores de cada poço $(\mathrm{n}=4)$

\begin{tabular}{lccc}
\hline $\begin{array}{l}\text { Concentração } \\
\mu g \mathrm{~L}^{-1} \text { de P }\end{array}$ & $\mathrm{R}$ & $\mathrm{G}$ & $\mathrm{B}$ \\
\hline 500 & $131 \pm 4,9$ & $134 \pm 4,7$ & $144 \pm 7,7$ \\
1000 & $95 \pm 7,3$ & $110 \pm 5,3$ & $136 \pm 3,1$ \\
1500 & $75 \pm 6,2$ & $94 \pm 3,9$ & $129 \pm 2,0$ \\
2000 & $45 \pm 6,2$ & $75 \pm 3,9$ & $120 \pm 2,5$ \\
2501 & $30 \pm 10,0$ & $69 \pm 6,0$ & $119 \pm 2,8$ \\
\hline
\end{tabular}

As curvas analíticas obtidas utilizando as equações 3 a 6 são mostradas na Figura 7, todas as curvas analíticas se mostraram lineares com um coeficiente de correlação $\left(\mathrm{R}^{2}\right)$ maior que 0.96 . Devido à pequena variação de $\mathrm{B}$ em função da concentração de fosfato, o coeficiente angular da Equação 5 foi pequeno $\left(4 \times 10^{-5}\right)$. Por sua vez,

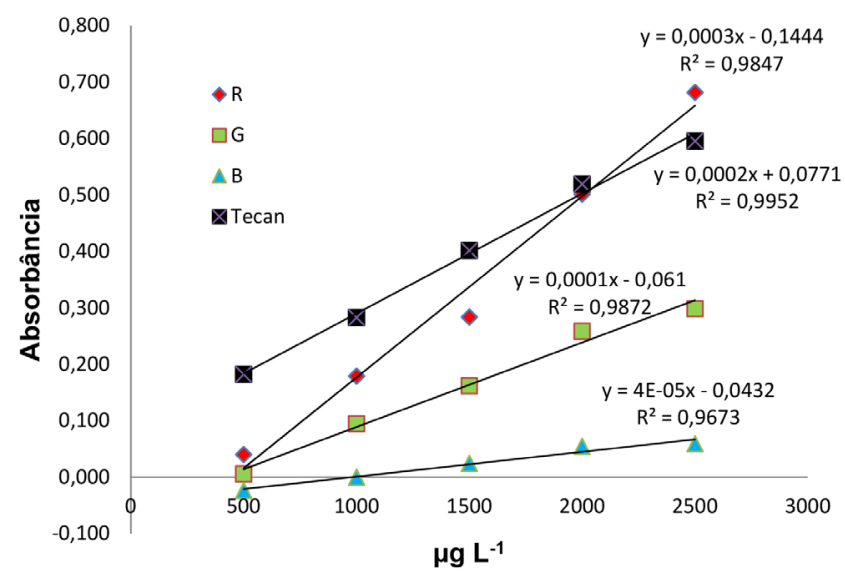

Figura 7. Concentração de fósforo ( $\left.\mu g L^{-1}\right)$ vs Absorbância. R: Equação 3; G: Equação 4 e B: Equação 5. como os valores de $\mathrm{R}$ variam mais que os valores de $\mathrm{G}$ em função da concentração de fosfato, a Equação 3 tem um coeficiente angular $\left(3 \times 10^{-4}\right)$ três vezes maior que a Equação $4\left(1 \times 10^{-4}\right)$.

A curva analítica obtida com o leitor de microplacas (Tecan) apresentou maior linearidade que as curvas obtidas com os valores de RGB extraídos das imagens digitais e um coeficiente angular $\left(2 \times 10^{-4}\right)$ menor que o coeficiente angular obtido com a equação 3 .

A curva analítica obtida com a Equação 6 se mostrou linear (Figura 8). A equação 6 foi utilizada, porque os valores de R, G e B variaram em função da concentração de fosfato. Desta forma, esta equação leva em consideração os valores dos três canais. Por exemplo, Moraes et al. ${ }^{27}$ determinaram a concentração de $\mathrm{Fe}^{2+}$, em um estudo da taxa de corrosão do ferro na água do mar, utilizando imagens digitais. Neste estudo, a absorbância foi calculada levando em conta a variação dos canais $\mathrm{G}$ e B. Em razão do complexo vermelho, formado entre o $\mathrm{Fe}^{2+}$ e a fenantrolina, apresentar pouca variação no canal $\mathrm{R}$ em função da concentração, os canais $\mathrm{G}$ e B apresentaram variações apreciáveis em função da concentração de $\mathrm{Fe}^{2+}$ na solução.

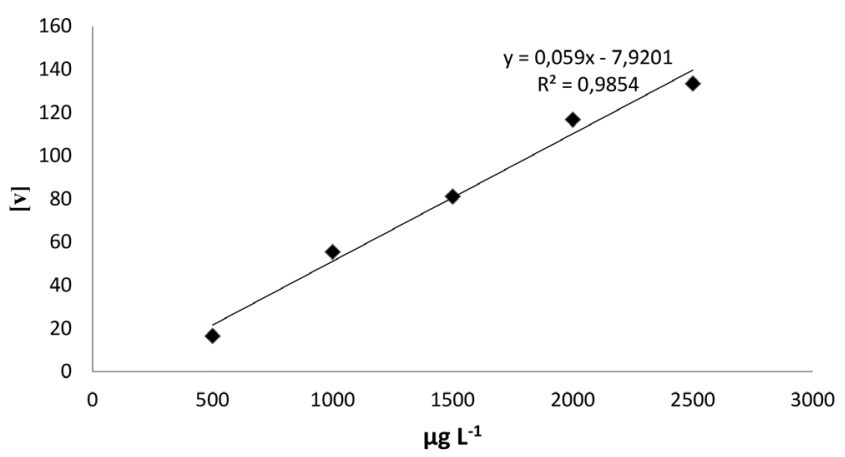

Figura 8. Curva de calibração obtida com a Equação 6 [v] vs concentração de fosfato

Oito microplacas foram preparadas e analisadas em $650 \mathrm{~nm}$, com um leitor de microplacas. Posteriormente, sete destas microplacas foram colocadas em um scanner frente a um fundo branco e as imagens obtidas em 300 dpi no formato JPEG. Na Tabela 2 está apresentado a média e o desvio padrão $(\mathrm{m} \pm \mathrm{s})$, as concentrações obtidas com as Equação 3, Equação 4 e Equação 6 apresentaram valores próximos dos obtidos com o leitor de microplacas. A Equação 5 não foi utilizada, porque apresenta um coeficiente angular muito pequeno (Figura 7). As amostras de refrigerante nomeadas como 50 significam que o refrigerante foi diluído na proporção 1:50, enquanto a nomeação 100 significam que o refrigerante foi diluído na proporção 1:100.

Utilizando um leitor de microplacas, o desvio padrão realtivo da concentração de fosfato nos refrigerantes foi de $12 \%$ em média,

Tabela 2. Concentração de fosfato em refrigerante, concentração determinada como $\mu \mathrm{g}$ de $\mathrm{P} \mathrm{L}^{-1}$. análises feitas utilizando um leitor de microplacas em $650 \mathrm{~nm}$ (650) $n=8$, concentrações de fosfato determinadas utilizando as imagens digitais e as Equação 3 (R), Equação 4 (G) e Equação 6 ([v]).n = 7

\begin{tabular}{lcccc}
\hline & 650 & $\mathrm{R}$ & $\mathrm{G}$ & {$[\mathrm{v}]$} \\
\hline Pepsi 100 & $146927 \pm 17291$ & $133826 \pm 21315$ & $133475 \pm 35777$ & $133475 \pm 35777$ \\
Pepsi 50 & $129631 \pm 13660$ & $130038 \pm 17921$ & $123247 \pm 25240$ & $123247 \pm 25240$ \\
Coca Zero 100 & $125952 \pm 25998$ & $112497 \pm 27633$ & $105558 \pm 43310$ & $105558 \pm 43310$ \\
Coca Zero 50 & $114048 \pm 19376$ & $93614 \pm 24017$ & $93507 \pm 29527$ & $93507 \pm 29527$ \\
Coca 100 & $180561 \pm 14565$ & $157117 \pm 29997$ & $157206 \pm 43673$ & $157206 \pm 43673$ \\
Coca 50 & $144687 \pm 12934$ & $139019 \pm 30634$ & $127930 \pm 32679$ & $127930 \pm 32679$ \\
Black 100 & $183754 \pm 12882$ & $157451 \pm 40086$ & $163093 \pm 51779$ & $163093 \pm 51779$ \\
Black 50 & $142768 \pm 16281$ & $131655 \pm 42857$ & $120089 \pm 39123$ & $120089 \pm 39123$ \\
\hline
\end{tabular}


enquanto o desvio padrão relativo obtido com as imagens digitais ficou entre $23 \%$ e $30 \%$. Por sua vez, o desvio padrão relativo obtido com a Equação 3 e Equação 4 foi de $23 \%$ e o desvio padrão relativo obtido com a Equação 6 foi de 30\%. Portanto, optou-se por fazer a determinação da concentração de fosfato nos refrigerantes utilizando-se a Equação 3.

Na execução da prática com alunos, os mesmos deverão escolher qual equação é mais adequada. Repare que na Tabela 2 o desvio padrão obtido com a Equação 4 é muito próximo do desvio padrão obtido com a Equação 6.

As concentrações obtidas com o leitor de microplacas $(n=8)$ e com a equação $3(n=7)$ foram comparadas, inicialmente, utilizando o teste F para comparar as variâncias. Os valores de F obtidos para os refrigerantes estão mostrados na Tabela 3. Como é mostrado na Tabela 3, as variâncias obtidas com o leitor de microplacas e com a Equação 3, em um intervalo de confiança de 95, não podem serem presumidas equivalentes para os refrigerantes: Guaraná Black 100 e Guaraná Black 50, visto que o valor de F crítico para 7 e 6 graus de liberdade foi de 4,2 .

Tabela 3. Variância $\left(\mathrm{s}^{2}\right)$, F calculado, t de Student calculado, 650 representa os valores obtidos com o leitor de micro placas a $650 \mathrm{~nm}$ e R representa os valores obtidos com a Equação 3. O valor tabelado para F, considerando um intervalo de confiança de $95 \%$, com 7 e 6 graus de liberdade é 4,2 . O valor tabelado de t para 13 graus de liberdade é de 2,16

\begin{tabular}{lcccc}
\hline & $\mathrm{s}^{2} 650$ & $\mathrm{~s}^{2} \mathrm{R}$ & $\mathrm{F}$ & $\mathrm{t}$ \\
\hline Pepsi 100 & $2,99 \mathrm{E}+08$ & $4,54 \mathrm{E}+08$ & 1,54 & 1,54 \\
Pepsi 50 & $1,87 \mathrm{E}+08$ & $3,21 \mathrm{E}+08$ & 1,19 & 1,19 \\
Coca Zero 100 & $6,76 \mathrm{E}+08$ & $7,64 \mathrm{E}+08$ & 1,55 & 1,55 \\
Coca Zero 50 & $3,75 \mathrm{E}+08$ & $5,77 \mathrm{E}+08$ & 1,25 & 1,25 \\
Coca 100 & $2,12 \mathrm{E}+08$ & $9,00 \mathrm{E}+08$ & 4,18 & 1,07 \\
Coca 50 & $1,67 \mathrm{E}+08$ & $9,38 \mathrm{E}+08$ & 3,65 & 3,65 \\
Black 100 & $1,66 \mathrm{E}+08$ & $1,61 \mathrm{E}+09$ & $5,41^{*}$ & 0,72 \\
Black 50 & $2,65 \mathrm{E}+08$ & $1,84 \mathrm{E}+09$ & $4,83^{*}$ & 0,77 \\
\hline
\end{tabular}

O teste t presumindo variâncias equivalentes mostrou que a CocaCola 50 apresenta concentração de fosfato diferente da obtida com o leitor de microplacas, porque o valor critico de $t$, em um intervalo de confiança de $95 \%$ para 13 graus de liberdade, é 2,16 . O teste $\mathrm{t}$ presumindo variâncias distintas para a concentração de fosfato no Guaraná Black 100 e Guaraná Black 50 mostra que não há diferença significativa entre os resultados obtidos com a Equação 3 e o leitor de microplacas, visto que o valor de t calculado é menor que o valor de $\mathrm{t}$ critico. Desta foram, as concentrações de fosfato nos refrigerantes determinadas com a Equação 3 não diferem dos valores obtidos com um leitor de microplacas, em um intervalo de confiança de $95 \%$, exceto para a Coca-Cola 50.
A Tabela 4 compara os valores obtidos com o leitor de microplacas com a Equação 4 e Equação 6. A comparação das concentrações de fosfato obtidas com um leitor de microplacas e com os valores obtidos com a Equação 4 são mostradas na Tabela 4 . O teste F mostra que as variâncias das concentrações de fosfato obtidas com a equação 4 são diferentes das variâncias obtidas com o leitor de microplacas para Pepsi 100, Coca-Cola 100 e Black 100.

O teste t presumindo variâncias equivalentes mostrou que não há diferença significativa entre os resultados obtidos com a Equação 4 e o leitor de microplacas, porque os valores de $\mathrm{t}$ calculados foram menores que o valor de $\mathrm{t}$ critico $(2,16)$.

$\mathrm{O}$ teste $\mathrm{t}$ presumindo variâncias diferentes também mostrou que não há diferença significativa entre os resultados obtidos com a Equação 4 e o leitor de microplacas, sendo que o t crítico ficou em torno de 2,36 .

A comparação das concentrações de fosfato obtidas com um leitor de microplacas e com os valores obtidos com a Equação 6 são mostradas na Tabela 4 . O teste $\mathrm{F}$ mostra que as variâncias das concentrações de fosfato obtidas com a Equação 3 são diferentes das variâncias obtidas com o leitor de microplacas para Coca-Cola 100 e Black 100.

$\mathrm{O}$ teste $\mathrm{t}$ presumindo variâncias equivalentes mostrou que há diferença significativa entre os resultados obtidos com a Equação 6 e o leitor de microplacas para a Coca-Cola 50, já que o valor de $\mathrm{t}$ calculado $(2,74)$ foi maior que o valor do t critico $(2,16)$.

$\mathrm{O}$ teste $\mathrm{t}$ presumindo variâncias diferentes mostrou que não há diferença significativa entre os resultados obtidos com a Equação 6 e o leitor de microplacas, sendo que o t crítico ficou em torno de 2,36.

$\mathrm{Na}$ Tabela 4, o teste t mostrou que as concentrações de fosfato obtidas com as Equação 3 e Equação 6 não apresentaram diferença significativa dos valores obtidos com o leitor de microplacas, exceto para Coca-Cola 50. Utilizando a Equação 6, não se observou diferença significativa com os valores obtidos com o leitor de microplacas para nenhum dos refrigerantes, embora esta equação forneça valores dedesvio padrão maiores que os obtidos com as outras equações. Isto indica que o método que utiliza imagens digitais fornece resultados similares aos obtidos com um leitor de microplacas.

É interessante que os alunos comparem os resultados obtidos com as imagens digitais (equações 3 a 6) com os resultados obtidos com um equipamento, como, por exemplo, um leitor de microplacas como foi feito neste trabalho. Provavelmente muitas instituições não tenham um leitor de microplacas, neste caso, os alunos podem fazer a quantificação de fosfato nos refrigerantes utilizando apenas as imagens digitais, e comparar os resultados obtidos com ambas as equações, bem como observar qual das equações fornece melhores resultados.

No desenvolvimento de um método analítico é interessante que o desvio padrão seja o menor possível. Desta forma, os alunos podem observar qual equação fornece o menor desvio padrão para cada refrigerante.

Tabela 4. Média (m), desvio padrão (s), variância $\left(\mathrm{s}^{2}\right)$. F e t calculados para a comparação dos valores obtidos com o leitor de microplacas a $650 \mathrm{~nm}$ com equação 4 (G) e com a equação 6 [v]

\begin{tabular}{|c|c|c|c|c|c|c|}
\hline & \multicolumn{3}{|c|}{$\mathrm{G}$} & \multicolumn{3}{|c|}{$[\mathrm{v}]$} \\
\hline & $\mathrm{s}^{2}$ & $\mathrm{~F}$ & $\mathrm{t}$ & $\mathrm{s}^{2}$ & $\mathrm{~F}$ & $\mathrm{t}$ \\
\hline Pepsi 100 & $1.28 \mathrm{E}+09$ & $4.34 *$ & 0.91 & $9.85 \mathrm{E}+08$ & 3.34 & 0.21 \\
\hline Pepsi 50 & $6.37 \mathrm{E}+08$ & 2.35 & 0.59 & $1.49 \mathrm{E}+08$ & 0.55 & 1.72 \\
\hline Coca Zero 100 & $1.88 \mathrm{E}+09$ & 3.80 & 1.17 & $1.83 \mathrm{E}+09$ & 3.71 & 0.18 \\
\hline Coca Zero 50 & $8.72 \mathrm{E}+08$ & 1.89 & 1.56 & $5.40 \mathrm{E}+08$ & 1.17 & 1.72 \\
\hline Coca 100 & $1.91 \mathrm{E}+09$ & $8.87^{*}$ & 1.35 & $1.22 \mathrm{E}+09$ & $5.67 *$ & 0.27 \\
\hline Coca 50 & $1.07 \mathrm{E}+09$ & 4.15 & 1.29 & $4.81 \mathrm{E}+08$ & 1.87 & 2.74 \\
\hline Black 100 & $2.68 \mathrm{E}+09$ & $9.02 *$ & 1.01 & $2.30 \mathrm{E}+09$ & $7.74 *$ & 0.14 \\
\hline Black 50 & $1.53 \mathrm{E}+09$ & 4.02 & 1.45 & $9.78 \mathrm{E}+08$ & 2.57 & 2.06 \\
\hline
\end{tabular}


De um ponto de vista didático é interessante que o aluno compare as concentrações de fosfato nos refrigerantes utilizando diluições de 1:50 e 1:100.

Em todos os refrigerantes, tanto com as imagens digitais (Equação 3, Equação 4 e Equação 6) como no leitor de microplacas, as concentrações obtidas com uma diluição de 1:50 foram menores que as concentrações obtidas com uma diluição de 1:100 (Tabela 2). Isto indica que uma maior diluição dos refrigerantes diminuí o efeito da matriz.

De maneira didática é interessante que os alunos utilizem as diluições (1:50 e 1:100) para observar o efeito do corante presente nos refrigerantes a base de cola. Quando este experimento foi realizado, as concentrações de fosfato obtidas utilizando as duas diluições foram comparadas utilizando-se o teste F para comparar as variâncias e o teste t para comparar as médias dos resultados.

Podemos comparar a variância da concentração de fosfato nos refrigerantes utilizando o teste $\mathrm{F}$, com base nas variâncias obtidas com a Equação 3 (Tabela 2 e Tabela 3). Os valores de F calculados para a Pepsi, Coca Zero, Coca-Cola e Guaraná Black são 1,41, 1,32, 1,04 e 1,14, respectivamente. Portanto, assumimos que não existe diferença significativa entre as variâncias obtidas com cada uma das diluições, visto que o valor de $\mathrm{F}$ tabelado, em um intervalo de confiança de $95 \%$, é de 3,78 .

Uma vez que variâncias equivalentes foram presumidas do teste $\mathrm{F}$, o teste $\mathrm{t}$ presumindo variâncias equivalentes foi utilizado para determinar os valores de t para Pepsi, Coca Zero, Coca-Cola e Guaraná Black de 0,36, 1,36, 1,11 e 1,16, respectivamente. Portanto, assumiu-se que não existe diferença significativa entre as concentrações determinadas com as diluições de 1:50 e 1:100, em um intervalo de confiança de $95 \%$.

\section{CONCLUSÃO}

A prática de ensino proposta introduz diversos conceitos primordiais ao ensino de ciências exatas e engenharias, como o uso de imagens digitais, a extração dos valores de RGB utilizando o plugin ReadPlate, exportação dos dados para o Microsoft Excel, construção de curvas analíticas, comparação de resultados utilizando testes estatísticos e introdução aos conceitos de espectrofotometria.

$\mathrm{O}$ uso do plugin ReadPlate no ImageJ permite que os valores de RGB sejam extraídos de uma microplaca de 96 poços em menos de 5 minutos. Por sua vez, a exportação dos dados do ImageJ para a tabela $1 \mathrm{~S}$ do Microsoft Excel permite que as curvas analíticas e a determinação da concentração de fosfato em cada refrigerante sejam determinadas imediatamente, após a aquisição das imagens digitais.

Os resultados obtidos com a equação 1 são equivalentes aos resultados obtidos com um leitor de microplacas, em um intervalo de confiança de $95 \%$, com exceção da Coca-Cola 50. Adicionalmente, a Equação 2 apresentou linearidade satisfatória $\left(R^{2}=0,98\right)$ e coeficiente angular maior que o obtido com um leitor de microplacas.

A prática de ensino proposta é simples e de baixo custo, em que se utiliza $50 \mu \mathrm{L}$ de reagente misto por poço, o que significa um gasto de 2,6 $\mathrm{mL}$ por microplaca. É importante ressaltar que a maioria dos métodos colorimétricos está sendo adaptada para microplacas para reduzir o uso de solventes e também o volume de resíduos.

A prática pode ser realizada utilizando-se apenas um scanner de mesa ou, quando um leitor de microplacas estiver disponível, os dois podem ser utilizados e os resultados comparados.

\section{AGRADECIMENTOS}

Os autores agradecem as bolsas e auxilio financeiro da FAPESC (Fundação de Amparo a Pesquisa do Estado de Santa Catarina), CNPq (Conselho Nacional de Desenvolvimento Científico e Tecnológico) e CAPES (Coordenação de Aperfeiçoamento de Pessoal de Nível Superior)

\section{REFERÊNCIAS}

1. Soldat, D. J.; Barak, P.; Lepore, B. J.; J. Chem. Educ. 2009, 86, 617.

2. Gomes, M. S.; Trevizan, L. C., Nóbrega, J. A.; Kamogawa, M. Y.; Quim. Nova 2008, 31, 1577.

3. Kohl, S. K.; Landmark, J. D.; Stickle, D. F.; J. Chem. Educ. 2006, 83, 644.

4. Cardoso, T. M. G.; Garcia, P. T.; Coltro, W. K. T.; Anal. Methods 2015, 7,7311 .

5. da Silva Lyra, W.; Sanches, F. A. C.; da Silva Cunha, F. A.; Diniz, P. H. G. D.; Lemos, S. G.; da Silva, E. C.; de Araujo, M. C. U.; Anal. Methods 2011, 3, 1975.

6. Diniz, P. H.; Dantas, H. V.; Melo, K. D.; Barbosa, M. F.; Harding, D. P.; Nascimento, E. C.; Araújo, M. C.; Anal. Methods 2012, 4, 2648.

7. dos Santos Benedetti, L. P.; dos Santos, V. B.; Silva, T. A.; Benedetti Filho, E.; Martins, V. L.; Fatibello-Filho, O.; Anal. Methods 2015, 7, 4138.

8. dos Santos Benedetti, L. P.; dos Santos, V. B.; Silva, T. A.; Benedetti-Filho, E.; Martins, V. L.; Fatibello-Filho, O.; Anal. Methods 2015, 7, 7568.

9. de Morais, C. D. L. M.; de Lima, K. M. G.; Anal. Methods 2015, 7, 6904.

10. Xia, M. L.; Wang, L.; Yang, Z. X.; Chen, H. Z.; Anal. Methods 2015, 7, 6654.

11. Fu, Q.; Wu, Z.; Li, X.; Yao, C.; Yu, S.; Xiao, W.; Tang, Y.; Biosens. Bioelectron. 2016, 81, 524 .

12. Santos, P. M.; Wentzell, P. D.; Pereira-Filho, E. R.; Food Anal. Methods 2012, 5, 89.

13. dos Santos, P. M.; Pereira-Filho, E. R.; Anal. Methods 2013, 5, 3669.

14. Christodouleas, D. C.; Nemiroski, A.; Kumar, A. A.; Whitesides, G. M.; Anal. Chem. 2015, 87, 9170.

15. Lozano-Calero, D.; Martín-Palomeque, P.; Madueño-Loriguillo, S.; J. Chem. Educ. 1996, 73, 1173.

16. Murphy, J.; J. Chem. Educ. 1983, 60, 420.

17. Nagul, E. A.; McKelvie, I. D.; Worsfold, P.; Kolev, S. D. Anal. Chim. Acta 2015, 890, 60.

18. https://pt.wikipedia.org/wiki/Bebida_de_cola. Acessada em fevereiro de 2017.

19. Caballero, T.; García, A. C.; Pascual, C.; Díaz, J. M.; Ojeda, A.; J. Invest. Allergol. Clin. Immunol. 1993, 3, 160.

20. Hadas, H. I; Hiller, N.; Guberman, D.; Am. J. Gastroenterol. 1993, 88, 127.

21. Wyshak, G.; Frisch, R. E.; Journal of Adolescent Health 1994, 15, 210.

22. Hernandez, A; Stampfer, M. J.; Ravnikar, V. A.; Willet, W. C.; Schiff, I.; Francis, M.; Longscope, C.; Mckinlay, S. M. Epidemiology 1993, 42, 128.

23. https://imagej.nih.gov/ij/. Acessada em fevereiro de 2017.

24. https://imagej.nih.gov/ij/plugins/readplate/index.html. Acessada em fevereiro de 2017.

25. https://drive.google.com/open?id=0B1vZRL3RwwcxNHQzZjdBMkpaSzQ. Acessada em fevereiro de 2017

26. Moraes, E. P.; da Silva, N. S.; de Morais, C. D. L.; Neves, L. S. D.; Lima, K. M. D.; J. Chem. Educ. 2014, 91, 1958.

27. Moraes, E. P.; Confessor, M. R.; Gasparotto, L. H. S.; J. Chem. Educ. 2015, 92, 1696

28. Damasceno, D.; Toledob, T. G.; Godinho, M. S.; da Silva, C. P.; de Oliveira, S. B.; de Oliveira, A. E.; Quim. Nova 2015, 38, 836.

29. Byoung-Yong, C.; Bull. Korean Chem. Soc. 2012, 33, 549. 